\title{
AMENAGEMENT DE LA CHUTE DE LAVEY (SUISSE) SUR LE RHONE
}

\author{
R. WALTHER \\ Ingénieur Civil E.P. Z
}

Nous rappelons, ci-après, les caractéristiques de ce nouvel aménagement hydroélectrique installé par la Ville de Lausame en remplacement d'une ancienne usine, celle dite du BoisNoir, à proximité de Saint-Maurice-en-Valais.

Le Rhòne, après avoir parcouru à faible pente, le palier d'alluvions qui forme le fond de la grande vallée jusqu'à Evionnaz, est repoussé contre la montagne par un cone d'alluvions, important qui détermine, jusqu'au droil de SaintMantice, tune chute denviron 40 mètres laquelle est actuellement utilisce par l'usine du Bois-Noit et le sera par celle de Lavey ultérieurement.

Géologiquement, le fond de la vallée présente deux ombilics (surcreusement glaciaire) successifs, le supérieur arrivant jusqu'au verrou très accusé du Pont de Saint-Maurice, à une profondeur allant jusqu'à $200 \mathrm{~m}$. ou $250 \mathrm{~m}$., sous les alluvions ainsi que l'ont démontré les professeurs Lugron et Oumanor par des mesures électriques. Le deuxième ombilic est celui du Lac Léman entre Saint-Maurice et Nyon, d'environ 300 à $350 \mathrm{~m}$. de profondeur, le « Lac do Genève » qui complète la nappe lémanique de Nyon à Genève étant d'origine glaciale récente et de faible profondeur.

A Evionnaz même, le rocher de la rive droite du Rhône est sensiblement vertical, ainsi que des sondages eflectués à travers l'alluvion jusqu'a $25 \mathrm{~m}$. de profondeur, an moyen de poutres en I l'ont démontré. Le barrage n'est done qu'appuyé sur le coteau rocheux, par sa culée rive droite at repose sur toute sa largeur d'environ $50 \mathrm{~m}$. sur le fond alluvionnaire qui se trouve formé d'une moraine argileuse compacte très favorable à l'étanchéité du barrage.

La chute de Lavey située, à part le barrage luimème en Valais, en territoire vaudois, dont Lausanne est la capitale est prévue pour un débit max. de $200 \mathrm{~m}^{3}$ qui correspond, sur la courbe des débits classés, à une tranche de 120 jours par an. L'éliage peut descendre assez bas. Pendant le dernier hiver 46-47, réputé tris sec, il a cté constaté un débil minimum de $40 \mathrm{~m} / \mathrm{sec}$ el, comme celui-ci élait pris entièrement par le camal d'amenée du Bois-Soir, il ne restail plus, dans le lit du Rhóne que 1 a $2 \mathrm{~m}^{2}$

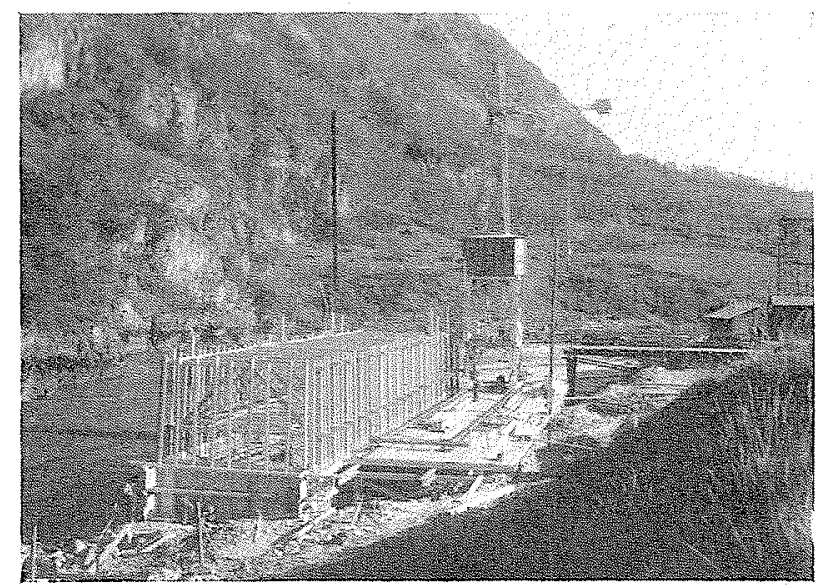

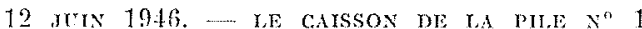
Ex CONSTRUCWON.

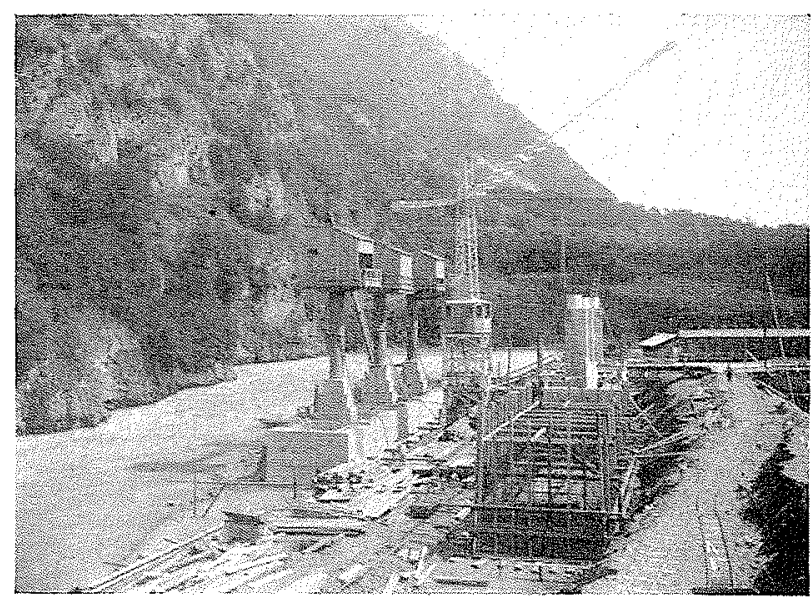

13 JUN 1946. - T.KS CAISSONS DE TA PILE $x^{\circ} 1$ ET DF T.A

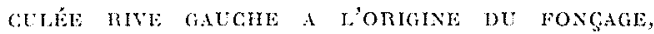
CELEL DU HUP RY ALE R. G, EN CONSTRLCTION 
d'infiltrations, ce qui dat bvitemment farorible a l'exécution des ourrages du barrage.

Enfin, comme débil maximum, on le considere comme normal jusqu'à $600 \mathrm{mb}$, te surplus jusqu'a 1000 et mème exceptionnellement $1200 \mathrm{~m} 3 / \mathrm{sec}$. ctant lobjet de plus-ralues contratoluelles accordces a lentrepreneur, pour ten: compte de l'augmentation des dilficultes d'execution, en particulier de la suprestom dans les caissons.

En principe, te niveau du Rhome, an droit du barrage, arami les lravaux, varail entre bos coles 436,80 à l'étiage el 41,50 aux H.E. normales. Par leftet des obstacles crés par la construction, le Rhone s'est afronillé, mais la cote des H.E. s'eleve, d'autre part, d'environ 1 mètre.

Il découle des indications ci-dessus, que la puissance maxima à altendre de cet aménagement est de lordre de $100.000 \mathrm{cr}(3$ turbines Kaplan de $33.000 \mathrm{cv}$ ou $75.000 \mathrm{kw}$ pouvane descendre pendant quelques jours dhiver a $12.500 \mathrm{kw}$, amélioration notable sur la puissaneo actuelle de f'Usine du Bois-Noir de $9.000 \mathrm{~km}$.

\section{TRAVAUX DU BARRAGE DU RHONE}

La seule méthode possible d'exécution des fondations du barrage - en particulier a cause de la présence de bloes de grande dimension dans les allurions - ctait lo foncage des piles par caissons à air comprimé. D'autre part, le mur en aile de la rive gauche, longeant le canal actuel de Bois-Noir, ne pouvait non plus itre fondé autrement, de même que le mur-guideau mitoyen a l'entrée du canal d'amenée de l'Usine. C'est don: a 19 caissons que l'on a recours au total :

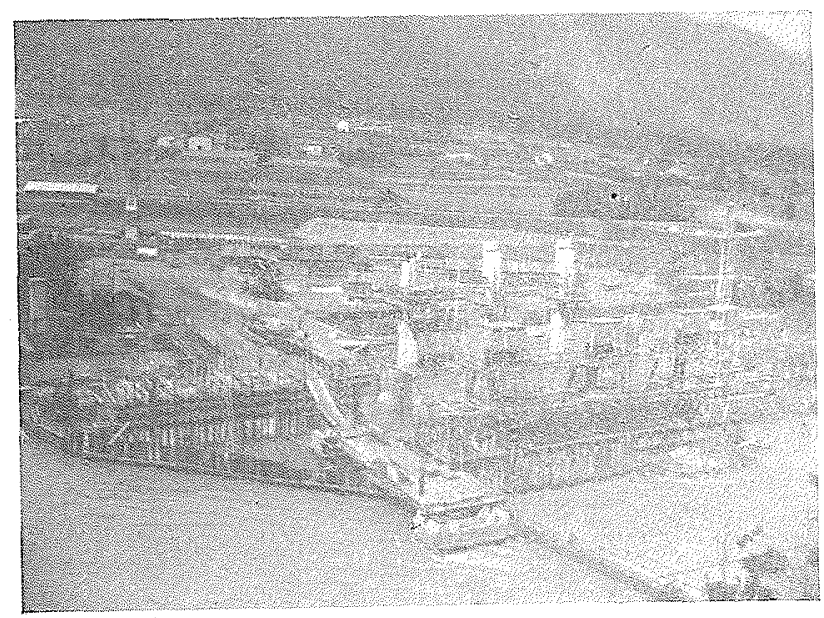

ETAT DES TRAVAUx AU 19 JUTHET 1946

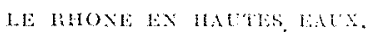

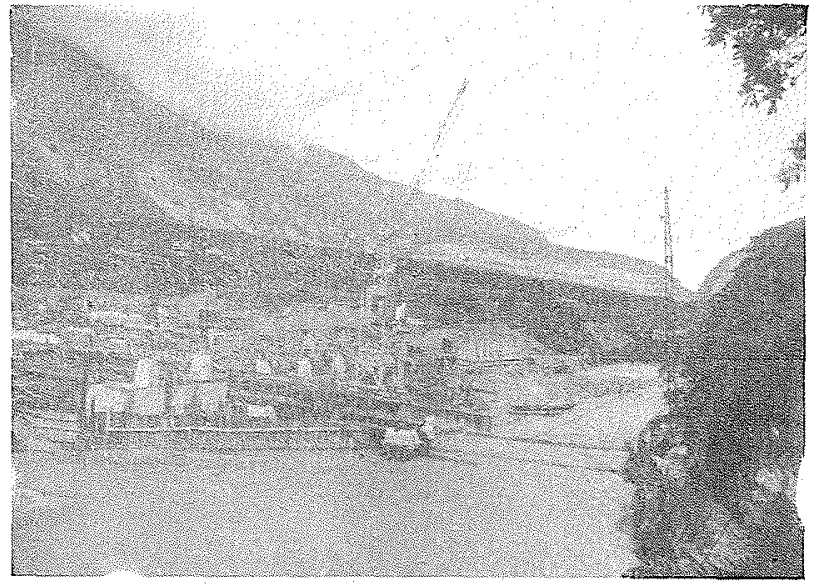

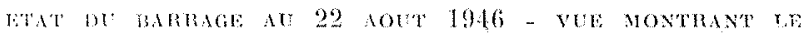

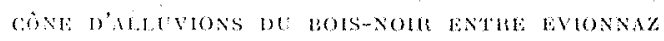
E' SAIN'MAURTCE.

3 grands de 221 et 207 m.c. de surface de base pour les 2 piles el la culée rive gauche, 10 moyens pour les murs et 6 petits pour les parafouilles des radiers des 3 passes.

Seuls ceux du barrage mème (les 3 grands et les 6 petiis) sont descendus jusqu’à la cote 421 $(422.50$ pour les pelits) soit à $14 \mathrm{~m} .(12 \mathrm{~m} .50)$ sous le niveau du radier des passes (435), les autres doivent arriver aux cotes 424 à 430 suivant leurs positions. On roit done que, suivant les cas, la pression peut aller jusqu'à $21 \mathrm{~m}$. ou meme un peu davantage ave les H.E. extraordinaires.

Le $1^{\text {rr }}$ caisson (C.1., pile 1) a été commencé le 15 avril 1946 el foncé à partir du 15 mai en 8 mois, avec 2 mois d'arrêt pour ferraillage et bétonnage de la pile. Ce n'est qu'au 25 janvier 1947 (fu'on put remplir la chambre de travail après nettoyage, mise en place des tuyaux pour l'injection à venir et retirer l'air comprimé, les sas et les cheminées.

II en lut de mòme du caisson de la culée rive gauche (C.O.), puis des caissons de rive attenant it celte culée (C.15 et C.16).

Le foncage du caisson de la pile 2(C.2) fut un peu plus rapide a cause de moins grandes sujéiions : 3 mois pour le ballage d'une enceinte de palplanches, un mois pour la construction du caision et 5 mois $1 / 2$ pour le foncage de $17 \mathrm{~m} .70$, la pression ayont atteint en H.E. (15 juillet) lo max. de $21 \mathrm{~m}$.

Les caissons ont été en général préparés sur terre-pleins à sec. Toutefois, celui de la pile 2 


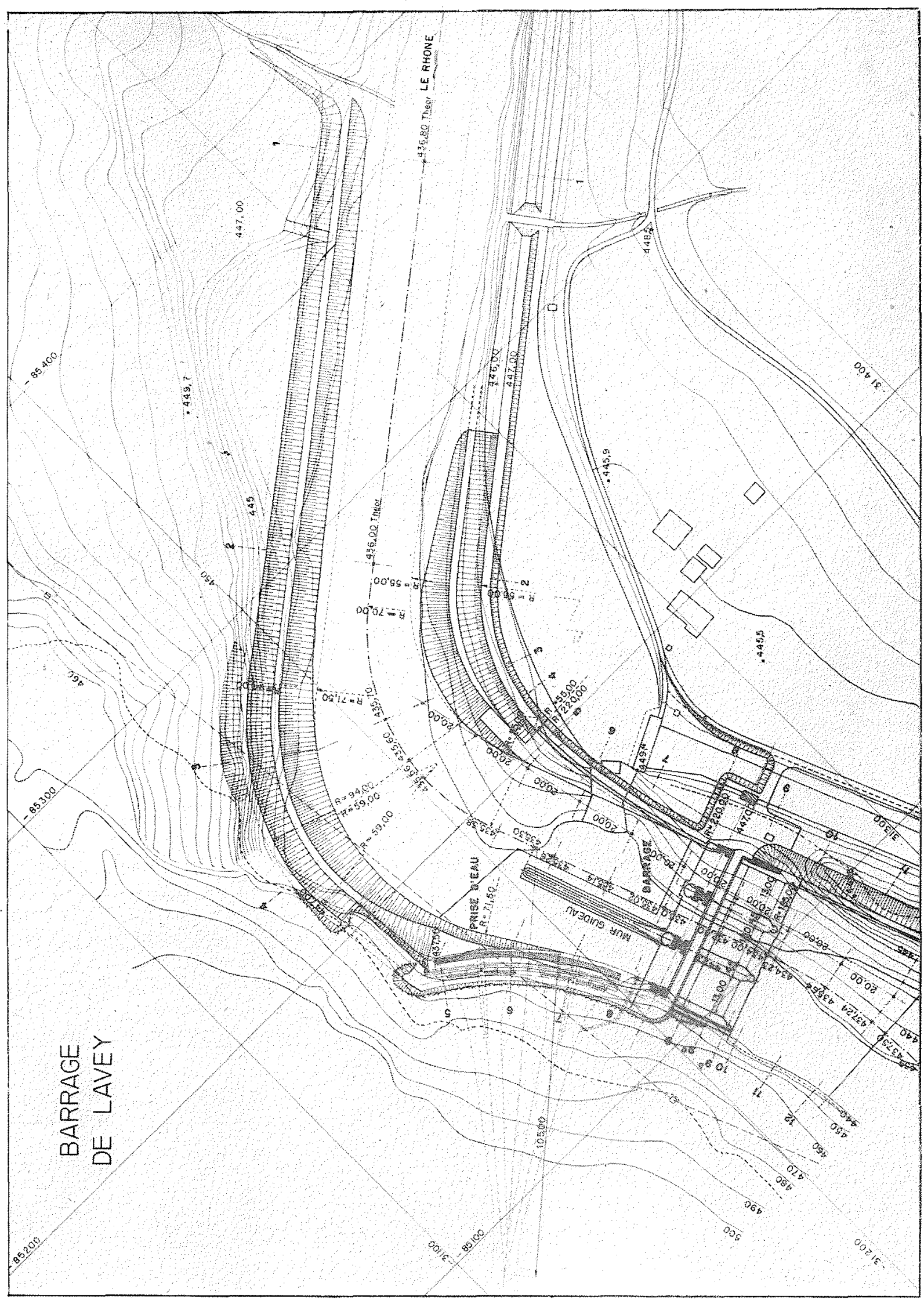




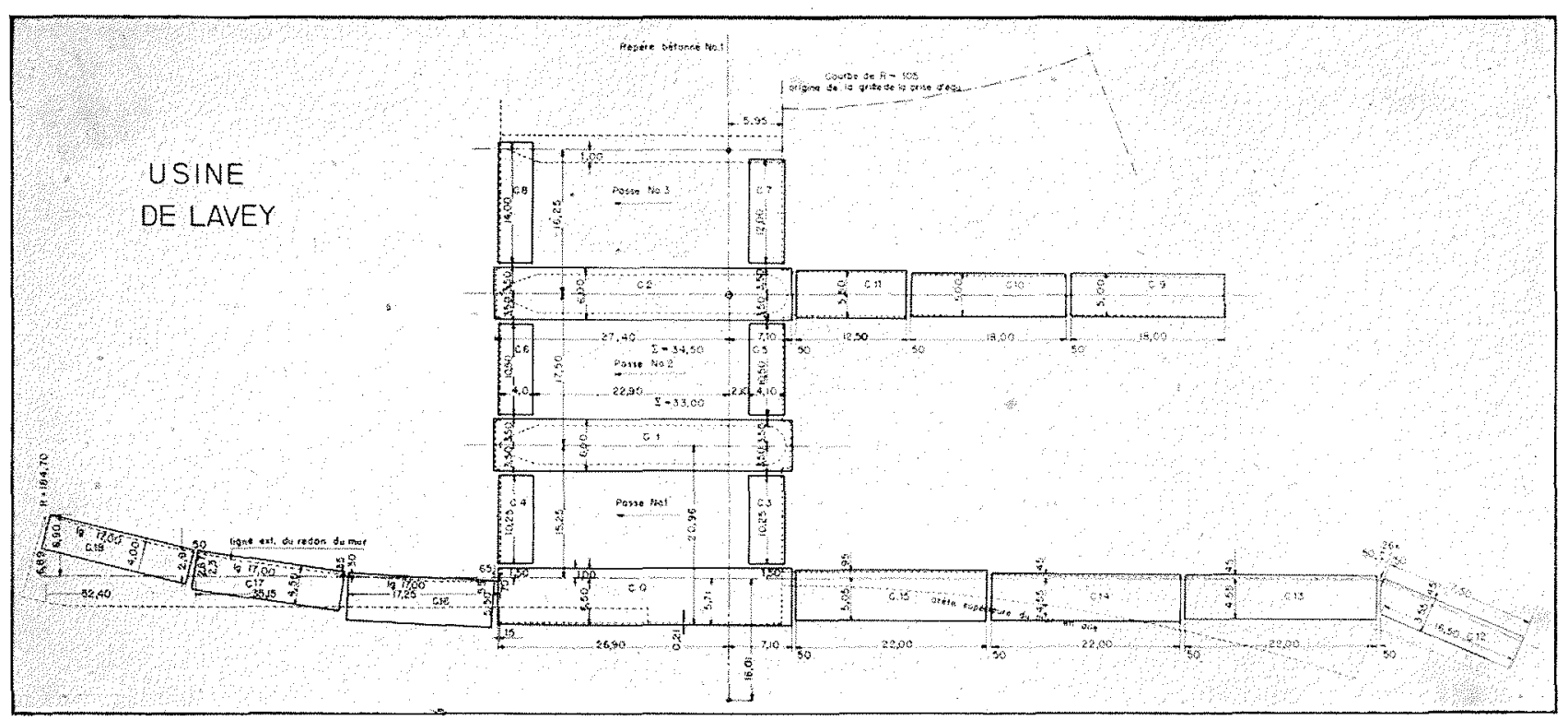

(C.2) fut construit dans l'enceinte de palplanches mentionnée plus haut, par pompage de quelques décimètres d'eau, de mème que ceux des parafoulles C.3 et C.4. Par contre, les 2 caissons parafouilles C.7 et C.8 de la $3^{6}$ passe (a droite) el de $60 \mathrm{~m}$.q. de surface de base, sont actuellemen! construits sur planches suspendues à des ponts métalliques à poutres paraboliques de 20 de portée et seront descendus à travers le courant dès la baisse des eaux en automne 1947. C'est là un mode de faire, peu habituel.

On peut constater que le fonçage est, en général, assez lent. Cela tient à plusieurs causes qu'il est intéressant de connaître :

Le sol d'alluvions à extraire des caissons s'est révélé plein de bloes de moraine ou d'éboulis de fort grande dimension; on arrivait à une proportion allant jusqu'à $30-40 \%$ de bloes de plus de $1 / 10^{e}$ de $\mathrm{m}^{3}$ sur le total extrait. Ces blows devaient naturellement être exploités à la mine et réduits en morceaux de faible dimension, pouvant passer par les sas à matériaux. Les mines elles-mèmes furent assez néfastes aux parois en consoles des chambres de travail, exícutées cependant en béton fortement armé ot cette circonstance incita a renforcer encore les caissons stubséquents.

Outre ces bloes, l'argile rencontrée au fond des fouilles fut un matérian extrèmement résistant et retarda la vitesse de foncage des derniers motres.

Il faut aussi dire que l'air comprimé à la fin des fonçages était aussi une cause de retard dans le rendement.
Une autre circonstance enfin retarda le fonçage et l'exécution générale du barrage, nous voulons parler du fait que lon a déc amené à relier par. des armatures formant poutre transversale, touts la largeur du barrage, en ménageant dans les piles en fonçage, des galeries ou viendront se déployer les armatures du radier. Réciproquement, des fers en attente, recourbés pour la descente des caissons; ont été mis en place dans les parois de ceux-ci, pour ètre recourbés dans le. radier. Ce ferrailage qu'on voit sur une de nos photos, demande naturellement un cofrage intérieur compliqué, de mème que celui des rainures des cadres des rannes et batardeaux.

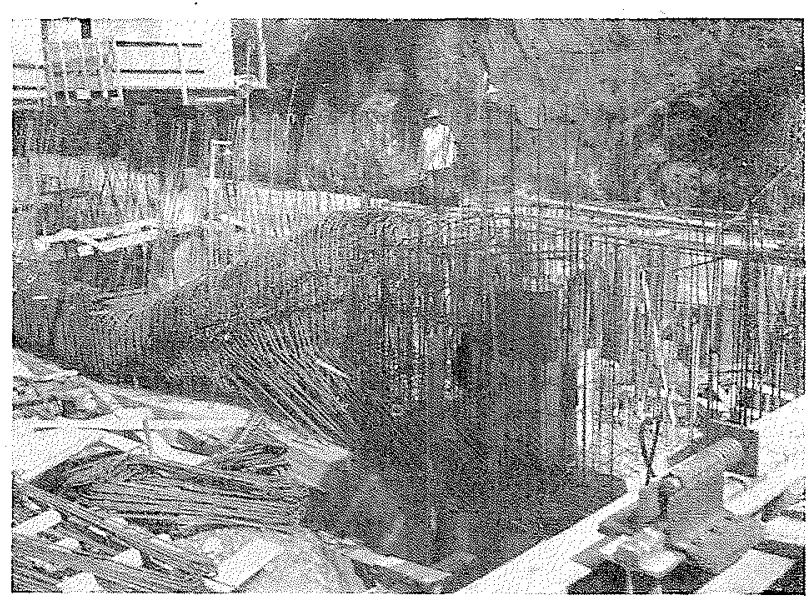

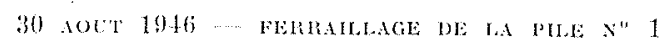

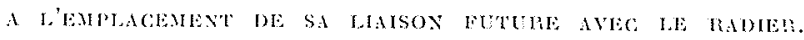




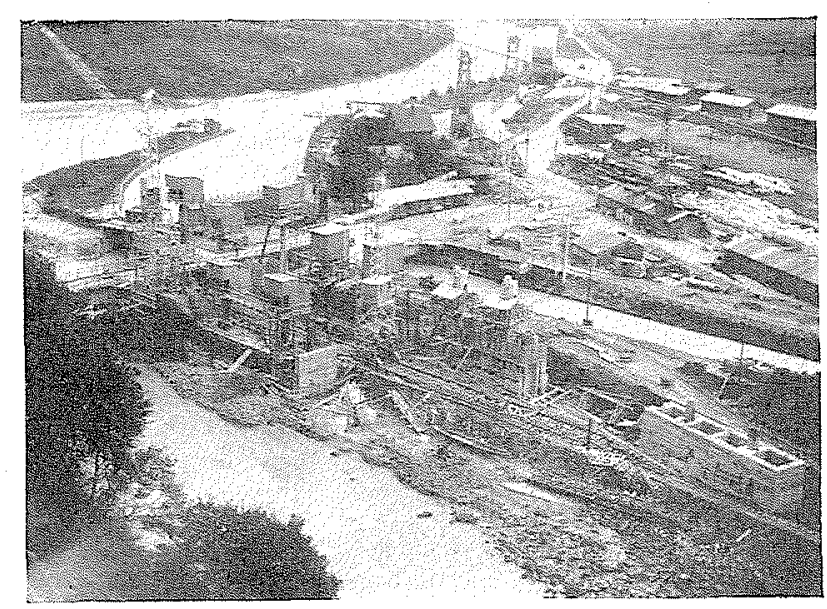

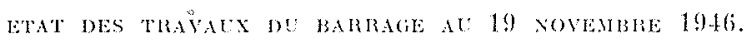

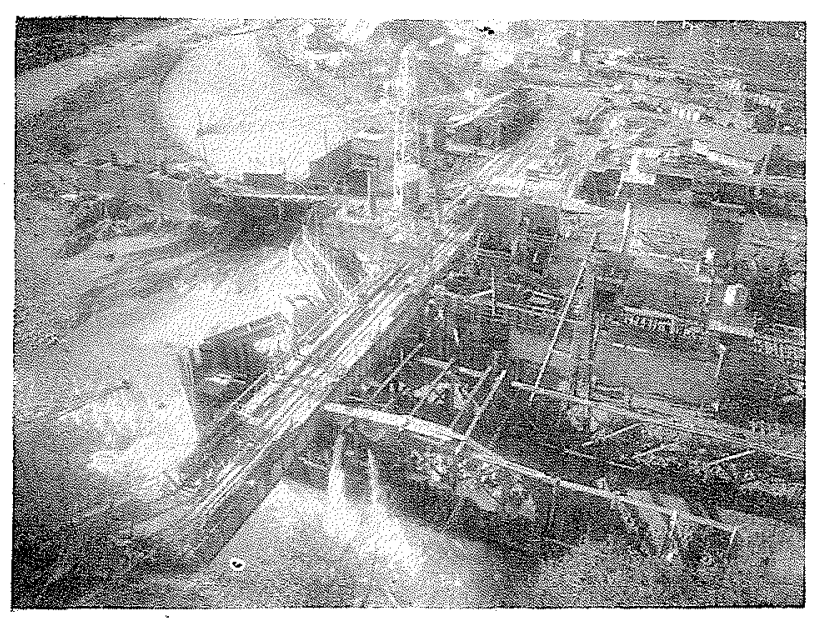

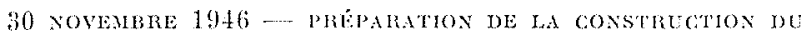
CAISSON DE LA PILE $x^{\circ} 2$ : EACEINTE DE PATPANCHES DANS LE HHONE AYES POAHAGE POER ASSÉCHEMENT DU TERRE-PHEL INTERTEUR.

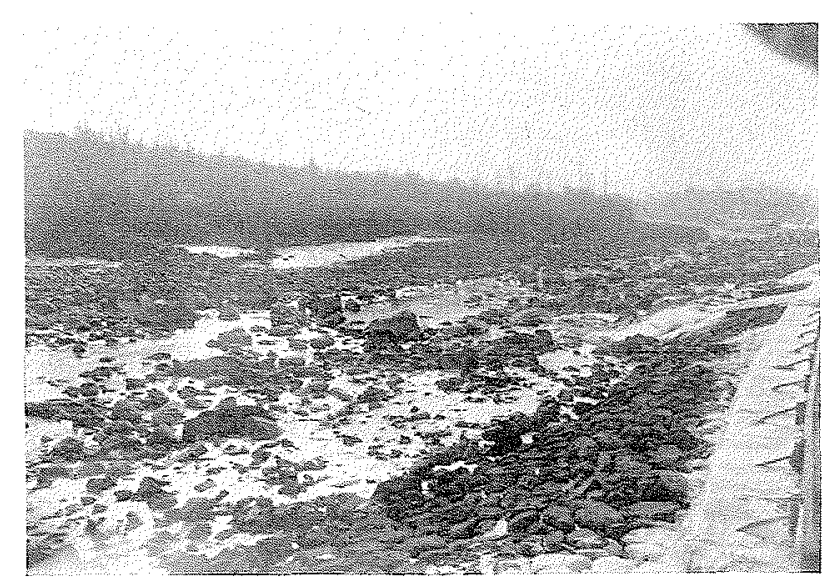

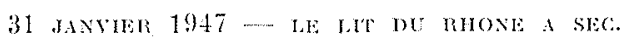

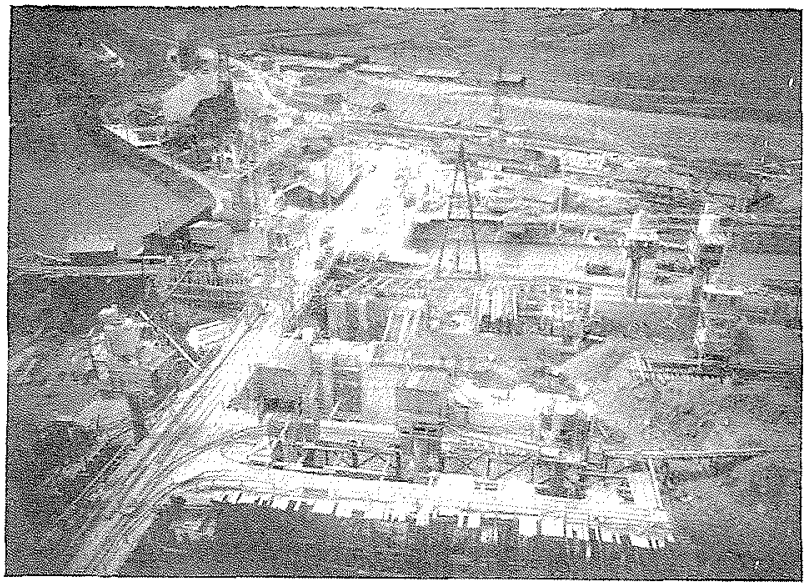

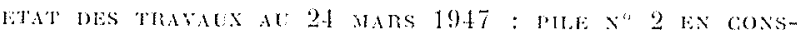

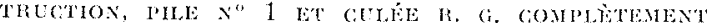

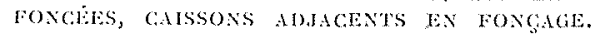

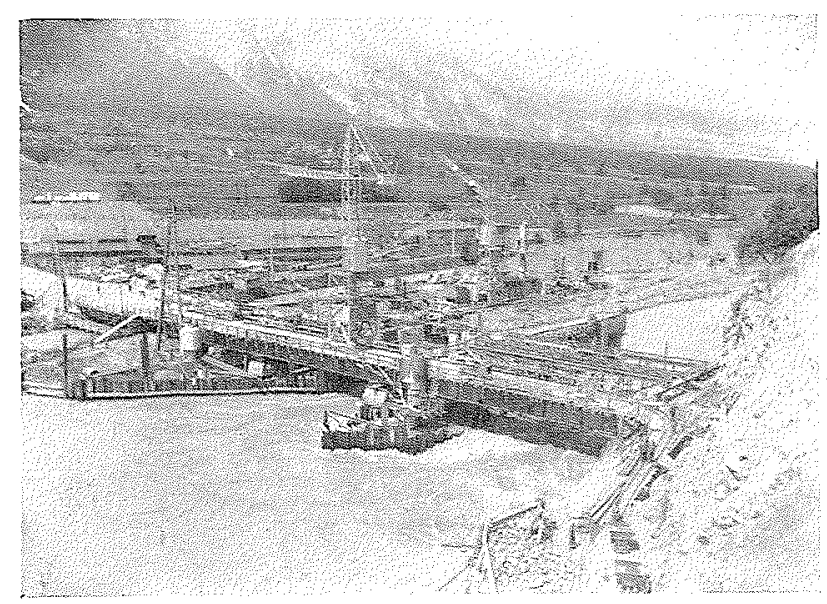

VUE De CHANTEL AY $1^{\mathrm{s}}$ AOUL 1947

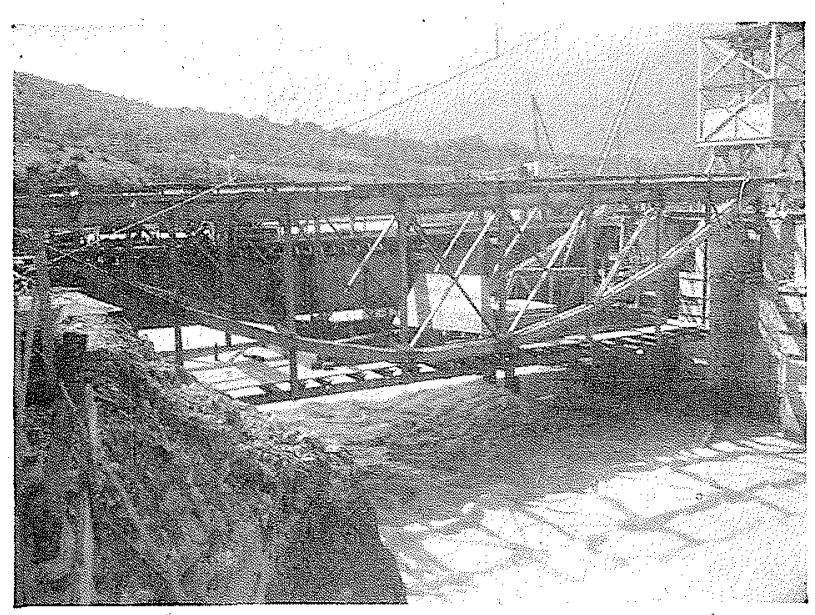

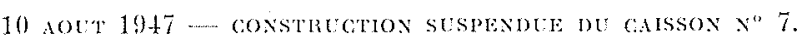




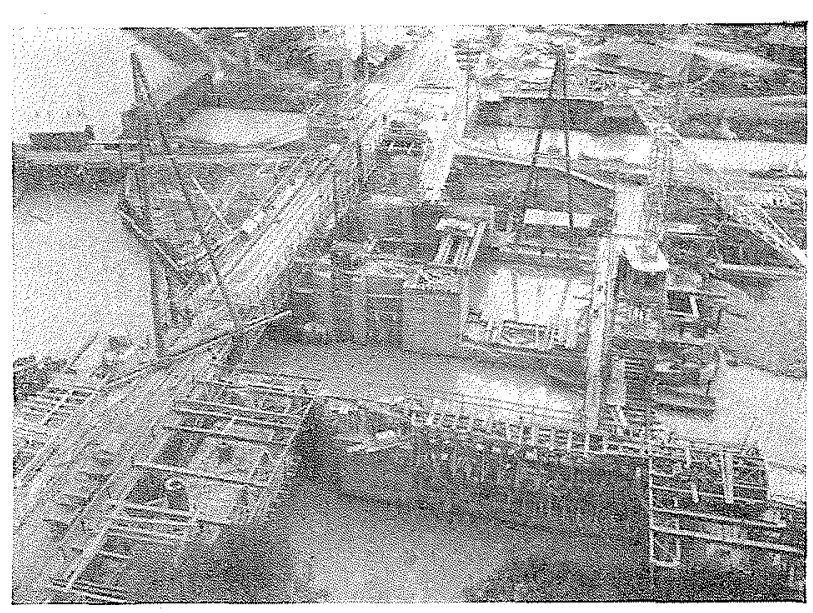

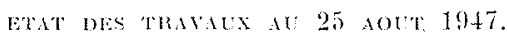

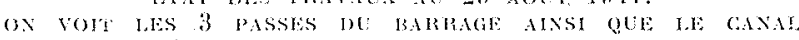

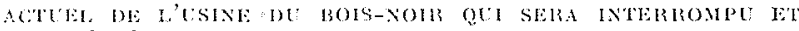

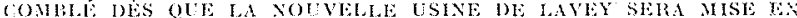

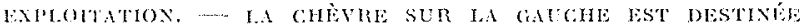

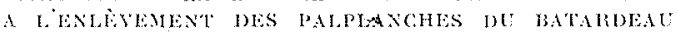
ENTOURAN LA PILE $x " 2$.

Une fois les deux caissons de la culée rive gauche et de la pile n" 1 descendus à fond, on a procédé au déblaiement de la passe à l'aide d'uns pelle mécanique et d'un pompage puissant derrière l'enceinte de palplanches qui l'entourait. Puis on a construit les deux petits caissons para- fouilles (C.3 et C.4) à l'amont et à l'aval du radier et on les a descendus rapidement de façon a créer le bassin où l'on ponra construire le radier lui-mème.

Avant cela, on va procéder à diverses opérations:

- l'injection clu fond de fouille des 4 caissons C.D. - C.1 - C.3 - C.4 (actuellement achevée).

- l'injection latérale des socles des piles et parafouiles en-dessous du niveau du radier.

- un drainage le long de la culée rive gauche, coté du terrain, pour éviter des pressions hydrauliques latérales, tant pendant l'aflouillement de la fondation du radier, que plus tard par la présence d'une nappe d'eau, une fois le barrage en eall.

- la mise en place provisoire d'une série do contreventements horizontaux, en gros bois ronds entre la culce el la pile.

Tous ces caissons sont reliés entre eux par des joints formés de palplanches métalliques enlevables après coup. De chaque coté d'un des caissons du radier, se trouvent ainsi 4 petites parois formant 2 fois 2 montants étanches, laissant. libre l'orifice des galeries intérieures des fer's de liaison. Sur la passe, on a donc 8 joints étanches asséchés par pompage, nettoyés, ferraillés puis bétonnés sous l'eau.

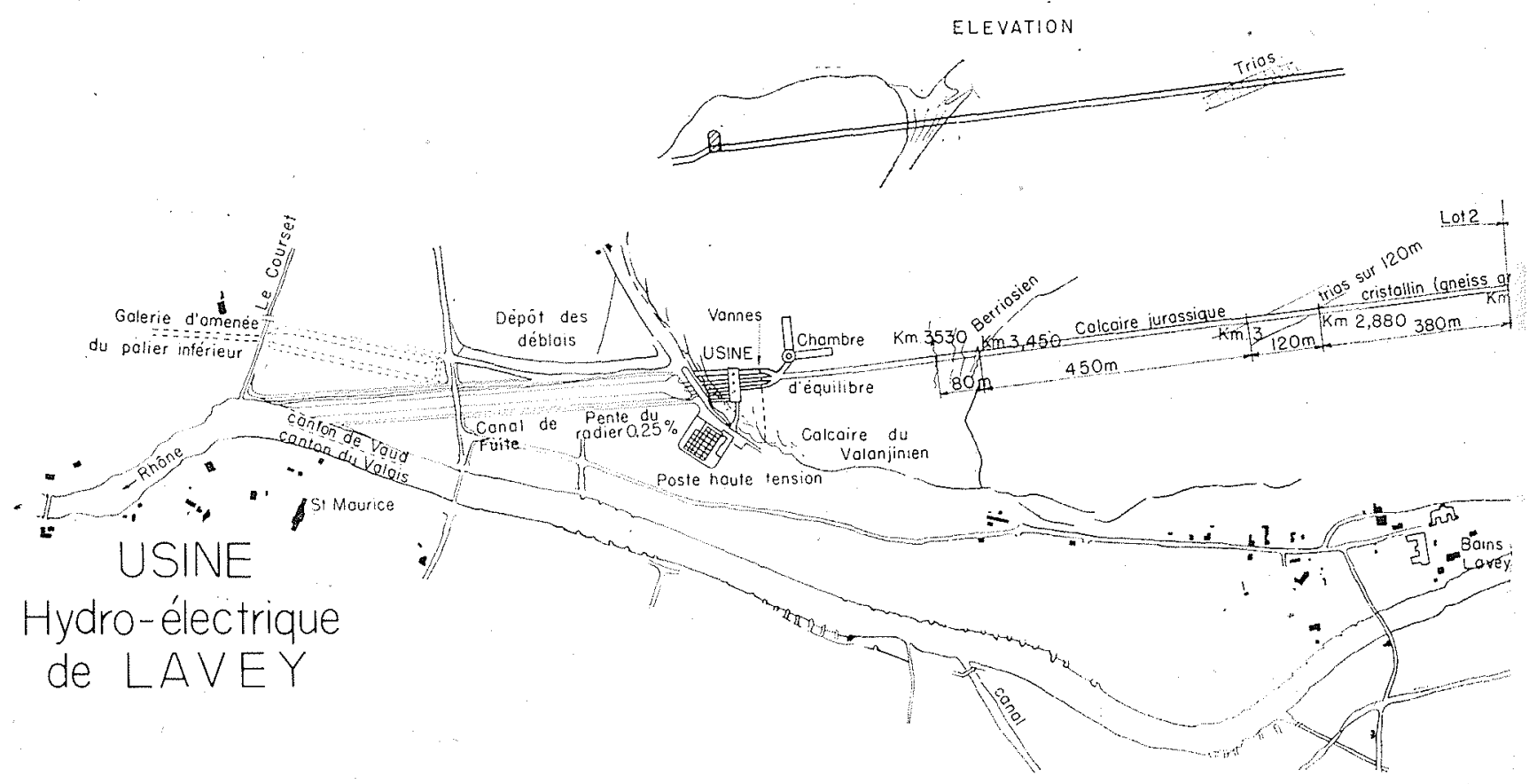


Un pompage général du radier permettra de construire celui-ci, des parois supplémentaires en palplanches sappuyant sur un rideau de parafouilles et empèchant les flots d'eau de pénétrer dans cette enceinte. L'exécution même du radier ne pourra d'ailleurs s'exécuter qu'aux eaux moyennes ou basses.

L'ensemble de ces travaux nécessite une attention constante et seule une entreprise spécialisée pouvait l'assurer de facon parfaite. C'esi le Consortium des Entreprises Zschokke ir Locher d'Aarau et Zurich, avec le concours des Maisons Oyex-Chessex de Lausanne et Curror ET Ronvir du Valais, par les soins duquel le barrage et la prise d'eau sont en cours d'exécution. L'ensemble de ces travaux devra ètre prèt pour la mise en eau de la chute dans le courant de l'automne 1949. Dès que le $1^{\text {rr }}$ groupe marchera a l'usine de Lavey, on interrompra la marche de l'usine du Bois-Noir, on libérera le Rhône du vieux barrage situé à $1 \mathrm{~km}$. en amont du nouveau barrage et le canal d'amenée actuel sera combli avec les déblais déposés en attente sur la rive gauche du fleuve.

Les vamnes du barrage ont $13 \mathrm{~m}$. de largeur par $11 \mathrm{~m}$. de hauteur. Elles sont formées de deux pelles à rouleaux, Ia supérieure déversante. Les vannes des deux passes de gauche seront, en principe, ouvertes pendant les hautes eaux, et évacueront les gros apports accumulés sur

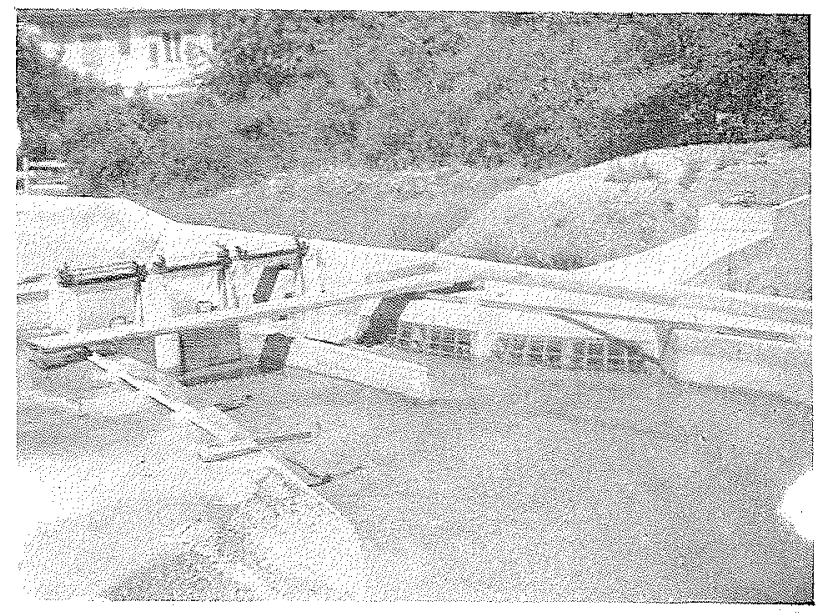

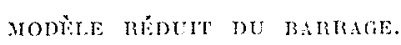

ceite partie du barrage, comme l'ont démontró les essais sur modèle réduit. La troisième passe ne sera ouverte qu'exceptionnellement.

Le sable entrant dans la galerie d'amence sera évacué par un dessableur sous pression et rejele au Rhône par un drain.

La galerie d'amenée de $4 \mathrm{~km}$., la chambre d'équilibre, l'usine souterraine et le canal de fuile onl eté adjugés au $1^{\circ}$ arril 1947 et sont

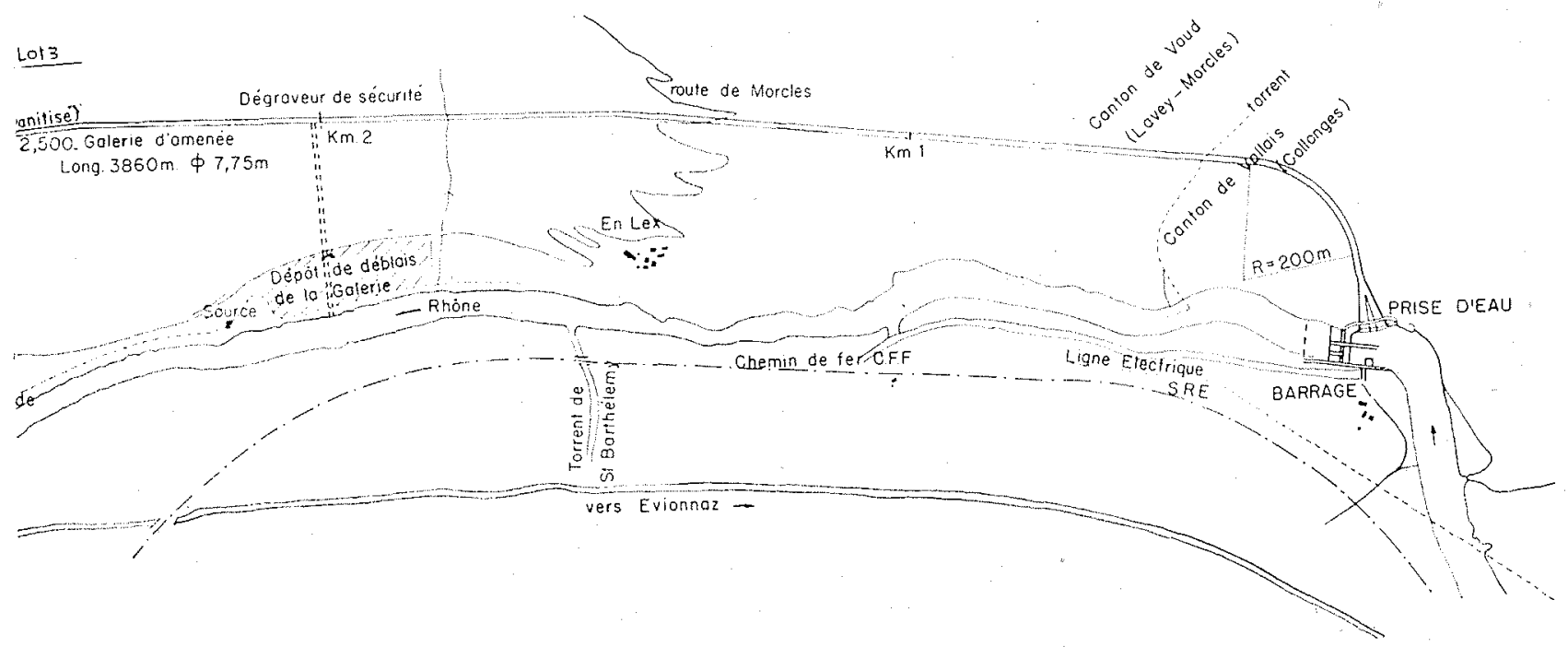


commencés sur toute la ligne. On prévoit des procédés rapides d'exécution et, le rocher paraissant sain, le délai indiqué plus haut pour la mise en marche de l'usine sera sans doute respectí. Nous reviendrons sur ces travax, quand ils seront en pleine activité.

Le Maître de l'ouvre, la Ville de Lausanne, est représenté pour cet important aménagement par le Directeur des Services Industriels, M. VON Drn As el celui des Services Electriques, M. MrystrR; et la Direction des travaux comprenant les études, les commandes et le contrôle est entre les mains des Mandataires, MM. Enser, Ing.-Conseil et Oacr, Prof. à l'Ecole d'Ingénieurs de Lausame.

(pholos R. Wallher).

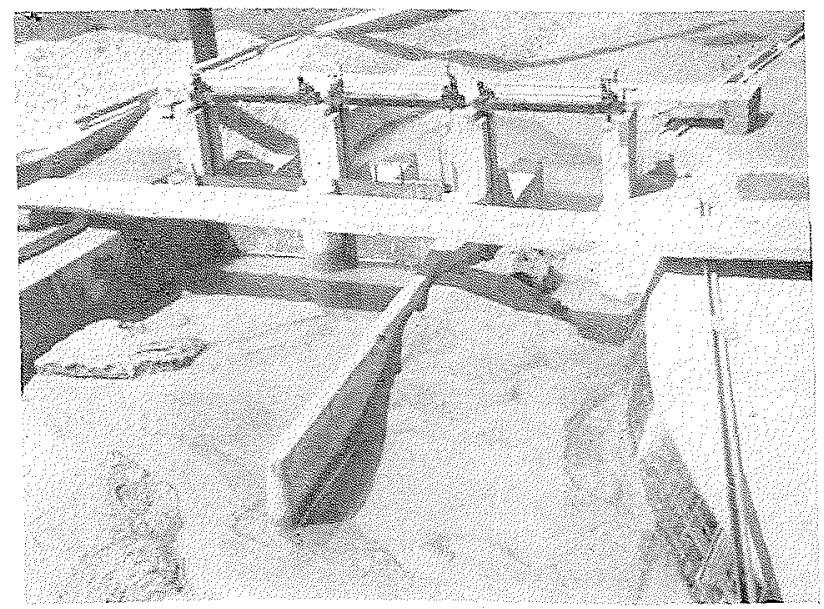

MODI:TE TEDETT DU BATRACE, 